\title{
The classification of minimal irregular surfaces of general type with $K^{2}=2 p_{g}$
}

\author{
Ciro Ciliberto, Margarida Mendes Lopes and Rita Pardini
}

\begin{abstract}
According to a result of O. Debarre from 1982 minimal irregular surfaces of general type satisfy $K^{2} \geqslant 2 p_{g}$. In this paper we classify those surfaces for which the equality $K^{2}=2 p_{g}$ holds.
\end{abstract}

\section{Introduction}

By a result of O. Debarre a minimal complex irregular surface $S$ of general type satisfies $K^{2} \geqslant 2 p_{g}$ ([Deb82, Théorème 6.1]). In this paper we complete the classification of the case $K^{2}=2 p_{g}$, which can be summarized as follows.

THEOREM 1.1. Let $S$ be a minimal complex surface of general type of irregularity $q>0$ satisfying $K^{2}=2 p_{g}$. Then $q \leqslant 4$.

If $q=1$, then the Albanese fibration is a genus two fibration with 2-connected fibres and $S$ is a double cover of a $\mathbb{P}^{1}$-bundle over the elliptic curve $\operatorname{Alb}(S)$.

If $q \geqslant 2$, then $\chi=1$ and:

(i) for $q=2$, the surface $S$ is the minimal desingularization of a double cover of a principally polarized abelian surface $(A, \Theta)$ branched over an effective divisor of class $2 \Theta$ with at most nonessential singularities;

(ii) for $q=3$, the surface $S$ is the symmetric product of a curve of genus three;

(iii) for $q=4$, the surface $S$ is the product of two curves of genus two.

This classification is the result of the work of many authors.

Debarre, besides establishing the inequality $K^{2}=2 p_{g}$ for minimal complex surfaces of general type of irregularity $q>0$, has shown that if equality holds, then $q \leqslant 4$, and that if $q=4$, then $S$ is the product of two curves of genus two ([Deb82, Théorème 6.3]).

For $q=1, K^{2}=2 p_{g}$ is the same as $K^{2}=2 \chi$. These surfaces had already been classified by Horikawa: their Albanese pencil has 2-connected genus two fibres, and the relative canonical map determines a $2-1$ cover of a $\mathbb{P}^{1}$-bundle over the elliptic curve $\operatorname{Alb}(S)$ (see [Hor82, Theorem 5.2] for a detailed description). We also note that in [Cat81] Catanese studied the case $q=\chi=1$.

Received 5 September 2013, accepted in final form 10 May 2014.

2010 Mathematics Subject Classification 14J29

This journal is (C) Foundation Compositio Mathematica 2014. This article is distributed with Open Access under the terms of the Creative Commons Attribution Non-Commercial License, which permits non-commercial reuse, distribution, and reproduction in any medium, provided that the original work is properly cited. For commercial re-use, please contact the Foundation Compositio Mathematica.

The first and the third author are members of G.N.S.A.G.A.-I.N.d.A.M. The second author is a member of the Center for Mathematical Analysis, Geometry and Dynamical Systems of Instituto Superior Técnico, Universidade de Lisboa. This research was partially supported by FCT (Portugal) through program POCTI/FEDER, Project PTDC/MAT/099275/2008 and Project PTDC/MAT-GEO/0675/2012, and by MIUR (Italy) through program PRIN 2010-2011 Geometria delle varietà algebriche. 


\section{Ciliberto, Mendes Lopes, Pardini}

Surfaces with $p_{g}=q=3$ and $K^{2}=6$ have been shown to be the symmetric product of a curve of genus three by Catanese and the first two authors ([CCM98, Prop. (3.22)]).

To obtain Theorem 1.1, we start by completing the classification of such irregular surfaces with $\chi=1$ (see $\S 3$ ). As explained above, the only yet unknown case was $p_{g}=q=2$. In Theorem 3.2, which is the main result in this paper, we show that these surfaces are exactly the so-called Catanese surfaces described in part (i) of Theorem 1.1. This had already been proved by Manetti in [Man03] under the extra assumption that $K$ be ample and by the first two authors in [CML02] under the assumption that the bicanonical map not be birational, but the lack of a complete description of this case has for many years been an annoying gap in the classification of surfaces with small invariants.

Surfaces with $p_{g}=q=2$ and $K^{2}=4$ are also interesting from the point of view of the Severi inequality $K^{2} \geqslant 4 \chi$ for minimal surfaces with Albanese dimension two, proven by Manetti in [Man03] for the case where $K$ is ample and then in general by the third author in [Par05]. Indeed, in [Man03] Manetti showed that for $K$ ample the Severi inequality is an equality exactly when $q=2$ and the Albanese map is a double cover of an abelian surface branched over a smooth curve $B$. Examples with $K$ non-ample and $K^{2}=4 \chi$ can be obtained by letting the branch curve $B$ acquire non-essential singularities and taking the minimal resolution of the double cover. It is natural to conjecture that these are the only surfaces with Albanese dimension two satisfying the equality in the Severi inequality. The present result proves the conjecture in the first case, that is, for $\chi=1$.

The new ingredient that allows us settle the case $p_{g}=q=2$, in a way that is in our view more conceptually transparent, is the recent classification in [CMP14] of curves $C$ with the following property: a Brill-Noether locus $W_{d}^{s}(C)$, properly contained in the Jacobian $J(C)$ of $C$, contains a variety $Z$ stable under translations by the elements of a positive dimensional abelian subvariety $A \subsetneq J(C)$ such that $\operatorname{dim}(Z)=d-\operatorname{dim}(A)-2 s$, that is, the maximum possible dimension for such $Z$ (see $\S 2.1$ and [CMP14]). Besides this, the proof relies, as usual in these matters, on two main tools: the paracanonical system and the Albanese map (see $\S 2.2$ ). In fact we prove that the general paracanonical curve $C$ is smooth of genus five and apply the results in [CMP14] to the Abelian surface $A=\mathrm{Pic}^{0}(S)$, which is contained in the Theta divisor of $J(C)$, to deduce that the Albanese map of $S$ has degree two. The result then follows by "classical" arguments.

Then we turn to the case $\chi>1, q \geqslant 2$. Since $q \leqslant 4$ and the case $q=4$ has already been described by Debarre, to complete the proof of Theorem 1.1 one needs to rule out the existence of minimal irregular surfaces with $K^{2}=2 p_{g}, 3 \geqslant q \geqslant 2, \chi>1$. This is done in $\S 4$, once again using "classical" arguments.

\section{Preliminaries}

2.1. Abelian subvarieties of Theta divisors of Jacobians. In [CMP14], following [AH91, DF93], we considered the following situation:

(*) $C$ is a smooth, projective, complex curve of genus $g, Z$ is an irreducible $r$-dimensional subvariety of a Brill-Noether locus $W_{d}^{s}(C) \subsetneq J^{d}(C)$, and $Z$ is stable under translations by the elements of an abelian subvariety $A \subsetneq J(C)$ of dimension $a>0$ (if so, we will say that $Z$ is A-stable).

Here $J^{d}(C)$ is the set of equivalence classes of divisors of degree $d$ on $C$ and $J(C):=J^{0}(C)$ 


\section{THE CLASSIFICATION OF MINIMAL IRREGULAR SURFACES...}

is the Jacobian variety of $C$. The Brill-Noether locus $W_{d}^{s}(C)$ is the subscheme of $J^{d}(C)$ corresponding to classes of divisors $D$ such that $h^{0}\left(\mathcal{O}_{C}(D)\right) \geqslant s+1$ and we set $W_{d}(C):=W_{d}^{0}(C)$ (see [ACGH85, Ch. IV]).

It was proved in [DF93] that if $\left(^{*}\right)$ holds, then $r+a+2 s \leqslant d$. In [CMP14], improving on partial results in [AH91, DF93], we give the full classification of the cases in which $\left(^{*}\right)$ holds and $r+a+2 s=d$. We will not need here the full strength of the results in [CMP14], only the part concerning the case $(d, s)=(g-1,0)$ (see [CMP14, Theorem 3.1]), which is as follows.

TheOrem 2.1. Let $C$ be a curve of genus $g$. Let $A \subsetneq J(C)$ be an abelian variety of dimension $a>0$ and let $Z \subset W_{g-1}(C)$ be an irreducible, $A$-stable variety of dimension $r=g-1-a$. Then there is a degree two morphism $\varphi: C \rightarrow C^{\prime}$, with $C^{\prime}$ smooth of genus $g^{\prime}$, such that one of the following occurs:

(a) we have $g^{\prime}=a, A=\varphi^{*}\left(J\left(C^{\prime}\right)\right)$ and $Z=W_{g-1-2 a}(C)+\varphi^{*}\left(J^{a}\left(C^{\prime}\right)\right)$;

(b) we have $g^{\prime}=a+1, r=a, \varphi$ is étale, $A$ is the Prym variety of $\varphi$, and $Z \subset W_{g-1}(C)$ is the connected component of $\varphi_{*}^{-1}\left(K_{C^{\prime}}\right)$ consisting of divisor classes $D$ with $h^{0}\left(C, \mathcal{O}_{C}(D)\right)$ odd, where $\varphi_{*}: J^{g-1}(C) \rightarrow J^{g-1}\left(C^{\prime}\right)$ is the norm map.

In particular, $Z \cong A$ is an abelian variety if and only if either we are in case (a) and $g=2 a+1$, or we are in case $(b)$.

2.2. Some generalities on irregular surfaces. Let $S$ be a smooth projective irreducible surface over $\mathbb{C}$. We will use the standard notation $q(S)=h^{1}\left(S, \mathcal{O}_{S}\right), p_{g}(S)=h^{0}\left(S, \Omega_{S}^{2}\right), \chi(S)=$ $\chi\left(\mathcal{O}_{S}\right)=p_{g}(S)-q(S)+1$ for the irregularity, the geometric genus, and the Euler characteristic of the structure sheaf, respectively. We will often use the simplified notation $p_{g}, q, \chi$. Note that, since we are working over the complex numbers, one has also $q=h^{0}\left(S, \Omega_{S}^{1}\right)$.

Numerical and linear equivalence will be denoted by $\sim$ and $\equiv$, respectively.

We will denote by $a: S \rightarrow \operatorname{Alb}(S)$ the Albanese morphism of $S$. The dimension of $a(S)$ is denoted by $\operatorname{Albdim}(S)$ and is called the Albanese dimension of $S$.

A pencil of genus $b$ on $S$ is a rational map $f: S \rightarrow B$ with connected fibres, with $B$ a smooth curve of genus $b$. The indeterminacy points of $f$ are the base points of the pencil. If $b>0$, the pencil is said to be irrational and has no base points.

An effective non-zero divisor $D$ on a smooth surface is said to be $m$-connected if for every decomposition $D=A+B$ with $A, B$ effective non-zero divisors one has $A \cdot B \geqslant m$.

A non-essential singularity of the branch locus $B$ of a double cover $X \rightarrow Y$ of surfaces, with $Y$ smooth and $X$ normal, is a double point or a triple point that has no infinitely near triple points (see [BPvdV04, Ch. III, Section 7], where these are called simple singularities).

As usual, we denote by $\left|K_{S}\right|$ (or simply $|K|$ ) the canonical system of $S$. If $\eta \in \operatorname{Pic}^{0}(S)$, the linear system $|K+\eta|$ is called a twisted canonical system of $S$. A curve $C_{\eta} \in|K+\eta|$ is a paracanonical curve on $S$.

Assume that $S$ is irregular of general type. We will denote by $\mathfrak{K}_{S}$ (or simply by $\mathfrak{K}$ ) the paracanonical system of $S$, that is, the Hilbert scheme of paracanonical curves on $S$. The morphism $p: \mathfrak{K} \rightarrow \operatorname{Pic}^{0}(S)$ is defined by $C \mapsto \mathcal{O}_{S}(C-K)$. As $\chi\left(\mathcal{O}_{S}\right) \geqslant 1$, there is a unique component $\mathcal{K}_{S}$ (or simply $\mathcal{K}$ ) of $\mathfrak{K}$ dominating $\operatorname{Pic}^{0}(S)$ via $p$. It is called the main paracanonical system of $S$.

If $\operatorname{Albdim}(S)=2$ and $\eta \in \operatorname{Pic}^{0}(S)$ is general, one has $h^{1}(S, \eta)=0$, hence $\operatorname{dim}(|K+\eta|)=\chi-1$ (this is the so-called generic vanishing theorem; see [GL87, Theorem 1]). If $\eta \in \operatorname{Pic}^{0}(S)$ and $C \in|K+\eta|$ are general, then $C$ corresponds to the general point of $\mathcal{K}$, which has dimension $q+\operatorname{dim}(|K+\eta|)=p_{g}$. 


\section{Ciliberto, Mendes Lopes, Pardini}

\section{Surfaces with $K^{2}=4$ and $p_{g}=q=2$}

Let $S$ be minimal, of general type, with $p_{g}=q=2$. One has $K^{2} \geqslant 4$ by [Deb82, Théorème 6.1 ] and the equality is attained in the following example.

Example 3.1. Let $(A, \Theta)$ be a principally polarized abelian surface. Let $p: S \rightarrow A$ be the double cover branched over an effective, smooth divisor $B$ in the class of $2 \Theta$, so that $p_{*} \mathcal{O}_{S}=\mathcal{O}_{A} \oplus$ $\mathcal{O}_{A}(-\theta)$, with $\theta$ in the class of $\Theta$ and $S$ smooth and minimal. Then $K=p^{*}(\theta)$ and the invariants of $S$ are $p_{g}=q=2, K^{2}=4$. We can also allow non-essential singularities on the divisor $B$. In that case $S$ will be the minimal resolution of the double cover of $A$ branched over $B$. One has $\operatorname{Alb}(S) \cong A$.

A special case is when $\Theta$ is reducible, that is, $A$ is the product of elliptic curves, and $\Theta=$ $E_{1}+E_{2}$, with $E_{i}$ elliptic curves such that $E_{1} \cdot E_{2}=1$. In that case $S$ has two elliptic pencils $\mathcal{M}_{1}, \mathcal{M}_{2}$ of curves of genus two (see [CML02, Example 7.1]; according to [Zuc03], this is the only case in which $S$ has an irrational pencil of curves of genus two; we will not use this result, though).

Note that the curves in $\mathcal{K}_{S}$ are the proper transforms via $p$ of the curves in the class of $\Theta$. If $\Theta$ is irreducible, then the general curve in $\mathcal{K}_{S}$ is smooth; otherwise, the general curve in $\mathcal{K}_{S}$ is the sum of a curve in $\mathcal{M}_{1}$ and a curve in $\mathcal{M}_{2}$.

In this section we prove the following classification result.

Theorem 3.2. A minimal surface of general type with $p_{g}=q=2, K^{2}=4$ is as in Example 3.1.

By [CML02, Proposition 2.3], minimal surfaces $S$ of general type with $K^{2}=4$ and $p_{g}=q=2$ have $\operatorname{Albdim}(S)=2$; that is, $a: S \rightarrow \operatorname{Alb}(S)$ is surjective. Surfaces of general type with $p_{g}=q=$ 2 and with an irrational pencil have been studied in [Pen11, Zuc03], but we will not use their results here.

Lemma 3.3. Let $S$ be a minimal surface of general type with $p_{g}=q=2, K^{2}=4$. Then

(i) either the general curve in the main paracanonical system $\mathcal{K}$ is smooth

(ii) or $S$ is as in Example 3.1, with $\Theta$ reducible.

Proof. Let $C \in \mathcal{K}$ be general. Write $C=F+M$, where $F$ is the fixed divisor of $\mathcal{K}$. Since $\operatorname{dim}(\mathcal{K})=2$, the curve $M$ moves in a two-dimensional system $\mathcal{M}$ of curves. First suppose that $M$ is reducible. Then $M$ must consist of two distinct irreducible components $M_{i}$, each moving in a one-dimensional family of curves $\mathcal{M}_{i}$, with $1 \leqslant i \leqslant 2$. The index theorem (see [BPvdV04, Ch. IV, Corollary 2.16]) yields $K \cdot M_{i} \geqslant 2$, for $1 \leqslant i \leqslant 2$. On the other hand,

$$
4=K^{2}=K \cdot F+K \cdot M_{1}+K \cdot M_{2},
$$

hence $K \cdot F=0, K \cdot M_{1}=K \cdot M_{2}=2$. Then $M_{1}^{2}=M_{2}^{2}=0$ and $\mathcal{M}_{i}$ is a pencil of curves of genus two, for $1 \leqslant i \leqslant 2$. Such a pencil is not rational by [Bea82, Lemme on p. 345], hence it is of genus one, because $q(S)=2$ and $\operatorname{Albdim}(S)=2$. This implies $M_{1} \cdot F=M_{2} \cdot F=0$, hence $F=0$ by the 2-connectedness of paracanonical divisors (see, for example, [BPvdV04, Ch. VII, Proposition 6.2]) and $M_{1} \cdot M_{2}=2$. Let $f_{i}: S \rightarrow E_{i}$ be the elliptic pencils $\mathcal{M}_{i}$, for $1 \leqslant i \leqslant 2$. The morphism $f=f_{1} \times f_{2}: S \rightarrow E_{1} \times E_{2}$ is a double cover and we are in case (ii).

Next we may assume that is $M$ irreducible, and we prove that $C$ is irreducible.

We claim that $M^{2} \geqslant 3$. In fact, by [CCM98, Lemma 0.14], $M^{2}=0$ would mean that $\mathcal{M}$ is a pencil and this is impossible because it has dimension two. Now, by [CCM98, Proposition 0.16], 


\section{The Classification of Minimal IRREGUlar SURFACES...}

a curve $M$ with $M^{2}=1$ moving in a two-dimensional system of curves varies in a linear system of rational curves and this is impossible because $S$ is of general type. Finally, $M^{2}=2$ is also excluded because, by [CCM98, Theorem 0.20], $S$ would be birational to a product of curves, which is not the case.

Suppose $F \neq 0$. Then by the 2 -connectedness of paracanonical divisors one has

$$
K^{2} \geqslant K \cdot M=F \cdot M+M^{2} \geqslant 5,
$$

a contradiction.

Finally, we prove that $C$ is smooth. Assume, to the contrary, that $C$ is singular. Let $c \in \mathcal{K}$ be the point corresponding to $C$. The two-dimensional tangent space $T_{\mathcal{K}, c}$ is contained in $H^{0}\left(C, N_{C \mid S}\right) \cong H^{0}\left(C, \mathcal{O}_{C}(C)\right)$, which is also two-dimensional, so that equality holds. It therefore corresponds to a $g_{4}^{1}$ on $C$. Every section of $H^{0}\left(C, \mathcal{O}_{C}(C)\right)$ in $T_{\mathcal{K}, c}$ vanishes at each singular point of $C$. This implies that $C$ has a unique singular point $x_{C}$. Consider the rational map $\operatorname{Pic}^{0}(S) \rightarrow S$ which associates with $C$ its singular point $x_{C}$. Since this map cannot be dominant, it follows that for $C$ general in $\mathcal{K}$ there is a one-dimensional system $\mathcal{K}_{C}$ of curves in $\mathcal{K}$ having, like $C$, a singular point at $x_{C}$. Since the curves in $\mathcal{K}_{C}$ have no variable intersection off $x_{C}$, the system $\mathcal{K}_{C}$ is a pencil, and it is rational because it has the base point $x_{C}$. Thus the surface $P$ parametrizing $\mathcal{K}$ would be ruled, a contradiction since $P$ is birational to $\operatorname{Pic}^{0}(S)$.

By Lemma 3.3 we may assume from now on that the general curve $C \in \mathcal{K}$ is smooth of genus five. Consider the restriction morphism $\mathfrak{r}: \operatorname{Pic}^{0}(S) \rightarrow J^{4}(C)$ sending $\eta$ to $\mathcal{O}_{C}(C+\eta)$. This map is injective by [CFM97, Proposition 1.6]. By generic vanishing, if $\eta \in \operatorname{Pic}^{0}(S)$ is general, one has

$$
h^{0}\left(C, O_{C}(C+\eta)\right)=h^{0}\left(S, O_{S}(C+\eta)\right)=1 .
$$

Hence the image $A$ of $\mathfrak{r}$ is an abelian surface contained in $W_{4}(C)$.

Lemma 3.4. In the above setup there exist a smooth genus two curve $C^{\prime}$ and a degree two morphism $\varphi: C \rightarrow C^{\prime}$ such that $A=\varphi^{*}\left(J^{2}\left(C^{\prime}\right)\right)$.

Proof. We can apply Theorem 2.1 with $g=5$ and $r=a=2$. Hence to prove the assertion it suffices to show that case (b) of Theorem 2.1 does not occur. Suppose that we are in case (b). Then, by Theorem 2.1 (cf. [BH04, $\S 12.6])$, for all $\eta \in \operatorname{Pic}^{0}(S)$, one has $h^{0}\left(C, L_{\eta}\right) \equiv 1(\bmod 2)$. On the other hand, $L_{0}=\mathcal{O}_{C}(C)$ and, by generic vanishing, $h^{0}\left(C, \mathcal{O}_{C}(C)\right)=2$, a contradiction.

End of the proof of Theorem 3.2. By Lemma 3.3, we may assume $C \in \mathcal{K}$ general to be smooth. By Lemma 3.4, there is a degree two morphism $\varphi: C \rightarrow C^{\prime}$, with $C^{\prime}$ of genus two, such that $A=\varphi^{*}\left(J^{2}\left(C^{\prime}\right)\right)$. Since $\varphi$ is ramified at four points, $A \cong J\left(C^{\prime}\right)$ is a principally polarized abelian surface. So $\operatorname{Pic}^{0}(S)$ is also principally polarized and we may identify both $\operatorname{Pic}^{0}(S)$ and $\operatorname{Alb}(S)$ with $A$.

Next, we prove that there is an involution $\mathfrak{i}$ of $S$ such that $\mathfrak{i}(C)=C$ and $\mathfrak{i}_{\mid C}$ coincides with the involution $\iota$ determined by the double cover $\varphi: C \rightarrow C^{\prime}$ (then $\mathfrak{i}$ will be biregular, since $S$ is minimal of general type). To define $\mathfrak{i}$ we argue as follows.

The curve $C$ is smooth by Lemma 3.3; let $x \in C$ be general and let $\mathcal{K}_{x}$ be the family of curves in $\mathcal{K}$ containing $x$. We claim that the set $\{x, \iota(x)\}$ coincides with the base locus $B_{x}$ of $\mathcal{K}_{x}$, and therefore is independent of $C$. Indeed, by the generality of $x$, there exists a $D \in \mathcal{K}_{x}$ such that $h^{0}\left(C, \mathcal{O}_{C}(D)\right)=1$ and the divisor $\left.D\right|_{C}$ is reduced and equal to $x+\iota(x)+y+\iota(y)$ for some $y \in C$. Since $B_{x}$ is invariant under $\iota$ by construction, we have two possibilities:

$$
\text { (a) } B_{x}=\{x, \iota(x), y, \iota(y)\} \quad \text { or } \quad \text { (b) } B_{x}=\{x, \iota(x)\} .
$$




\section{Ciliberto, Mendes Lopes, Pardini}

In case (a) all the curves of $\mathcal{K}_{x}$ would cut the same divisor on $C$. Since the map $\operatorname{Pic}^{0}(C) \rightarrow \operatorname{Pic}^{0}(S)$ is injective, this would mean that all the curves of $\mathcal{K}_{x}$ are linearly equivalent, which goes against the assumption that $C$ be general.

Consider the minimal desingularization $X$ of the quotient $S / \mathfrak{i}$. It contains a two-dimensional family of (generically smooth and irreducible) curves $\Gamma$ of genus two, with $\Gamma^{2}=2$, that is, the genus two quotients of the curves in $\mathcal{K}_{S}$ under the involution i. By [CCM98, Theorem 0.20], all curves $\Gamma$ are isomorphic and $X$ is birational to the symmetric product $\Gamma(2)$, which is birational to $J(\Gamma)$. So we have a degree two map $S \rightarrow J(\Gamma)$, which is in fact a morphism since $J(\Gamma)$ is an abelian variety and $S$ is smooth. The universal property of the Albanese map immediately implies that $A=J(\Gamma)$ and that $S \rightarrow J(\Gamma)$ is the Albanese morphism $a: S \rightarrow A$. Note that via $X \rightarrow A$ the curves $\Gamma$ are mapped to $\Theta$-divisors, hence the paracanonical curves are mapped 2-to-1 to $\Theta$-divisors by $a$.

Let $B$ be the branch divisor of $a: S \rightarrow A$. Since the curves in $\mathcal{K}$ have genus five, we have $B \cdot \Theta=4$. By the index theorem we have $B^{2} \leqslant 8$. On the other hand, $B$ is divisible by 2 in the Neron-Severi group $N S(A)$, hence $B^{2} \geqslant 8$. In conclusion, $B^{2}=8$ and the index theorem implies that the class of $B$ is $2 \Theta$ and the assertion follows.

Remark 3.5. Let $S$ be as in Example 3.1 with $B$ smooth. Then the ramification curve $R$ is a canonical curve isomorphic to $B$, hence smooth. So the general curve $C \in|K|$ is smooth and, as above, we can consider the restriction map $\operatorname{Pic}^{0}(S) \rightarrow W_{4}(C)$, whose image is an abelian surface $A \subset W_{4}(C)$. We can use Theorem 2.1, but now the argument of Lemma 3.4 does not apply because $C$ is not a paracanonical curve. In this situation the Prym case (b), and not case (a), of Theorem 2.1 occurs. Indeed, the bicanonical morphism is not birational for $S$ : it is in fact composed with an involution $\sigma$ such that $\Sigma=S / \sigma$ is a surface with 20 nodes (over which the double cover $\phi: S \rightarrow \Sigma$ is ramified). If $X$ is the minimal desingularization of $\Sigma$, one has $p_{g}(X)=2, q(X)=0, K_{X}^{2}=2$ (see [CML02, Proposition 6.5]). So the general curve $C \in\left|K_{S}\right|$ is the étale double cover of the general curve $C^{\prime} \in\left|K_{X}\right|$. One has the following commutative diagram:

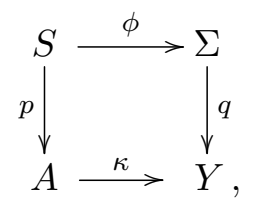

where $Y \subset \mathbb{P}^{3}$ is the 16-nodal Kummer surface of $A$ ( $\kappa$ is the obvious double cover), and $q: \Sigma \rightarrow Y$ is a double cover branched along a smooth plane section $H$ of $Y$ (which pulls back via $\kappa$ to the branch divisor of $p$ ), plus six nodes lying on a conic $\Gamma \subset Y$.

By [Bea88, Proposition 4], there is a unique curve $C_{0}$ in the intersection of $\left|K_{S}\right|$ with $\mathcal{K}$, that is, the proper transform of $\Gamma$ on $S$. It is interesting to notice that for $C_{0}$ both cases (a) and (b) of Theorem 2.1 occur at the same time (see [CMP14, Remark 3.2]).

We finally notice that the idea of the proof of Theorem 3.2 can also be applied to recover the classification of minimal surfaces $S$ with $p_{g}=q=3$ (see [CCM98, Proposition (3.22)]). In this case $q$ is odd, the analogue of Lemma 3.4 does not hold, and the Prym case (b) of Theorem 2.1 occurs. We do not dwell on this here. 


\section{THE CLASSIFICATION OF MINIMAL IRREGULAR SURFACES. .}

\section{Proof of Theorem 1.1}

As explained in the introduction, once we have Theorem 3.2, to prove Theorem 1.1, it suffices to show the following result.

Proposition 4.1. There is no minimal complex surface of general type $S$ with $2 \leqslant q \leqslant 3, \chi \geqslant 2$, and $K^{2}=2 p_{g}$.

Proof. Suppose by contradiction that such a surface $S$ exists.

Claim 4.2. One has $\operatorname{Albdim}(S)=2, q=3, p_{g}=4$.

Proof of Claim 4.2. Suppose, by contradiction, that $\operatorname{Albdim}(S)=1$, and let $a: S \rightarrow B$ be the Albanese pencil, with $B$ of genus $q$. We denote by $g$ the genus of the fibres of $a$; one has $g \geqslant 2$ because $S$ is of general type,

By [Hor82, Theorem 3.1], if $K^{2}<3 \chi$, the general fibre of $a$ is hyperelliptic of genus $g=2$ or 3. Since $B$ has genus $q$, applying the slope inequality for hyperelliptic fibrations ([Hor82, Theorem 2.1]), one has

$$
K^{2} \geqslant 4 \frac{g-1}{g}(\chi+(g+1)(q-1)) .
$$

From $K^{2}=2 p_{g}$, one concludes that $q=1$, a contradiction.

So $K^{2} \geqslant 3 \chi$. Then the inequality $2 p_{g}=K^{2} \geqslant 3 p_{g}-3 q+3$ yields $p_{g} \leqslant 6$ for $q=3$ and $p_{g} \leqslant 3$ for $q=2$. Since $K^{2}=2 p_{g}$, one has $K^{2} \leqslant 12$ if $q=3$ and $K^{2} \leqslant 6$ if $q=2$. But, by Arakelov's theorem (see [Bea82, Corollaire on p. 344]), we have

$$
K^{2} \geqslant 8(g-1)(q-1) \geqslant 8(q-1),
$$

a contradiction.

So $\operatorname{Albdim}(S)=2$. Then the Severi inequality proved in [Par05] gives $2 p_{g}=K^{2} \geqslant 4 \chi$, that is, $p_{g} \leqslant 2 q-2$. Since $\chi \geqslant 2$ and $q \leqslant 3$, the only possibility is $p_{g}=4, q=3$.

Claim 4.3. The surface $S$ has no irrational pencil $f: S \rightarrow B$ with $B$ of genus $b>1$. Therefore

(i) the map $\Phi: \bigwedge^{2} H^{0}\left(S, \Omega_{S}^{1}\right) \rightarrow H^{0}\left(S, \mathcal{O}_{S}\left(K_{S}\right)\right)$ is injective;

(ii) the Albanese image $\Xi$ of $S$ is not covered by elliptic curves.

Proof of Claim 4.3. Let $g$ be the genus of the general fibre of $f$. Again by Arakelov's theorem (see [Bea82, Corollaire on p. 344]) we have

$$
8=K^{2} \geqslant 8(g-1)(b-1),
$$

hence $g=b=2$. By [Hor77, Theorem 3], one has

$$
K^{2} \geqslant 2 \chi-6+6 b=10,
$$

a contradiction. This proves the first assertion and statement (i) follows by Castelnuovo-de Franchis' theorem ([dFr05], see [Bea78, Proposition X.9] for a modern reference) and the fact that, since $q=3$, all two-vectors are decomposable.

To prove statement (ii), suppose that $\Xi$ is covered by elliptic curves. Then there is an elliptic curve $E \subset \operatorname{Alb}(S)$ such that the image of $S$ via the composition of the Albanese morphism and the morphism $\operatorname{Alb}(S) \rightarrow \operatorname{Alb}(S) / E$ is a curve $B$. Since $B$ has to span $\operatorname{Alb}(S) / E$, which has dimension two, the curve $B$ has genus $b \geqslant 2$, contradicting the first assertion. 


\section{Ciliberto, Mendes Lopes, Pardini}

Claim 4.4. Let $F$ be the general fibre of a pencil $f: S \rightarrow B$ with $B$ of genus $b$ (possibly $b=0$ and the pencil is linear, with base points), and let $F$ be irreducible of geometric genus $g$. Then

(i) we have $K \cdot F \geqslant 4$ and $g \geqslant 3$;

(ii) if $K \cdot F=4$, then either $F^{2}=0, g=3, b=1$ or $F^{2}=2, b=0, g=4$ and $K \sim 2 F$.

In particular, $S$ has no pencil of curves of genus two and its bicanonical map is a birational morphism.

Proof of Claim 4.4. By Claim 4.3, we have $b \leqslant 1$. By blowing up if necessary, we may assume that the pencil has no base points and that $F$ is smooth of genus $g$. Then, by [Bea82, Lemme on p. 345], one has $3=q \leqslant b+g$ with equality only if $S$ is birational to a product of curves of genus $b$ and $g$. Since $S$ is of general type and $b \leqslant 1$, equality cannot hold and thus either $b=1$ and $g \geqslant 3$ or $b=0$ and $g \geqslant 4$.

Now consider the original surface $S$ again.

If $K \cdot F<4$, then by the index theorem and the fact that, by the adjunction formula, $K \cdot F$ and $F^{2}=0$ have the same parity, one has either $F^{2}=0, K \cdot F=2$ or $F^{2}=1, K \cdot F=3$. The former case implies $g=2$, a contradiction. The latter case gives $g=3$ and $b=0$ because the pencil has a base point, again a contradiction. Hence $K \cdot F \geqslant 4$. If equality holds, again by the index theorem and parity, either $F^{2}=0$ or $F^{2}=2$ and $K_{S} \sim 2 F$. In the former case, $g=3$ and thus $b=1$.

The bicanonical map is a morphism, as it always is for a minimal surface of general type with $p_{g} \geqslant 1$ (see [BPvdV04, Ch. VII, Theorem 7.4]). Since we have just proved that $S$ has no genus two pencil, by [CCM98, Theorem A] the bicanonical morphism of $S$ is birational.

Finally, we prove Proposition 4.1 by showing that surfaces $S$ as in Claim 4.2 do not exist. Suppose otherwise. Consider the map $\phi: S \rightarrow \mathbb{P}^{2}$ associated with the linear system $|\operatorname{Im}(\Phi)|$ (see Claim 4.3(i)). A standard local computation shows that this is the composition of the Albanese map $a: S \rightarrow \Xi$ with the Gauss map $\gamma: \Xi-\rightarrow \mathbb{P}^{2}$ which maps a general point $x \in \Xi$ to the point of $\mathbb{P}\left(T_{\mathrm{Alb}(S), 0}\right)^{*} \cong \mathbb{P}^{2}$ corresponding to the line $\mathbb{P}\left(T_{\Xi, x}\right)$ translated to the origin of $\operatorname{Alb}(S)$. The map $\gamma$ is dominant. Otherwise the line bundle determined by $\Xi$ would not be positive definite (see [BH04, Proposition 4.4.2]) and therefore $\Xi$ would be covered by elliptic curves, which is not the case. Hence the image of the canonical map $\varphi: S \rightarrow \mathbb{P}^{3}$ is a surface $\Sigma$.

Consider the multiplication map

$$
\mu: \operatorname{Sym}^{2}\left(H^{0}\left(S, \mathcal{O}_{S}(K)\right)\right) \rightarrow H^{0}\left(S, \mathcal{O}_{S}(2 K)\right) .
$$

Since both spaces have dimension 10, we have two possibilities:

(i) The map $\mu$ is an isomorphism; since the bicanonical map of $S$ is a birational morphism (see Claim 4.4), the map $\varphi$ is then also a birational morphism.

(ii) We have $\operatorname{dim}(\operatorname{ker}(\mu))=1$; in this case $\Sigma$ is a quadric.

Since $q$ is odd and $\operatorname{Albdim}(S)=2$, the canonical system is contained in the main paracanonical system $\mathcal{K}$ (see [MPP13, Theorem 1.3]). Hence if the general canonical curve is irreducible or smooth, then the general curve in $\mathcal{K}$ is also irreducible or smooth, respectively.

Assume that we are in case (i). Since $\varphi$ is a morphism, the general canonical curve is smooth. Let $C \in \mathcal{K}$ be general. Then by the above remark $C$ is also smooth. For any paracanonical curve $D \in|2 K-C|$ there exists a quadric $Q_{D}$ of $\mathbb{P}^{3}$ such that $D+C$ is the divisor of $\varphi^{*}\left(Q_{D}\right)$. Since $h^{0}\left(S, \mathcal{O}_{S}(D)\right)=\chi=2$, there exist at least two distinct quadrics $Q_{1}$ and $Q_{2}$ of $\mathbb{P}^{3}$ containing $\varphi(C)$. 


\section{THE CLASSIFICATION OF MINIMAL IRREGULAR SURFACES. . .}

Since $\varphi(C)$ is irreducible and non-degenerate of degree eight, this is a contradiction. So case (i) does not occur.

Assume now that we are in case (ii) and suppose first that $\Sigma$ is non-singular. The two line rulings $\left|L_{1}\right|,\left|L_{2}\right|$ of $\Sigma$ determine two pencils on $S$ with general fibres $F_{1}, F_{2}$, respectively. For $1 \leqslant i \leqslant 2$, the strict transform of a general element of $\left|L_{i}\right|$ is numerically equivalent to $r_{i} F_{i}$, with $r_{i} \geqslant 1$, and $K-\left(r_{1} F_{1}+r_{2} F_{2}\right)$ is numerically equivalent to an effective divisor. Hence we have

$$
8=K^{2} \geqslant r_{1} K \cdot F_{1}+r_{2} K \cdot F_{2} \geqslant 4\left(r_{1}+r_{2}\right)
$$

(the last inequality follows by Claim 4.4). So $r_{1}=r_{2}=1, K \cdot F_{1}=K \cdot F_{2}=4$ and, by Claim 4.4, $\left|F_{1}\right|$ and $\left|F_{2}\right|$ are distinct linear pencils with $F_{1}^{2}=F_{2}^{2}=F_{1} \cdot F_{2}=2$ (in particular, $F_{1} \sim F_{2}$ and $\left.K \sim 2 F_{1} \sim 2 F_{2}\right)$. The pencil $\left|F_{i}\right|$ has a base scheme $\beta_{i}$ of length two, hence $F_{i}$ is smooth of genus four, for $1 \leqslant i \leqslant 2$.

Set $F=F_{i}$ (with $i=1$ or 2$)$. Since $h^{1}\left(S, \mathcal{O}_{S}\left(K_{S}+F\right)\right)=0$ by Ramanujam's vanishing theorem (see [BPvdV04, Ch. IV, Theorem 12.5]), the restriction sequence

$$
0 \rightarrow \mathcal{O}_{S}\left(K_{S}\right) \rightarrow O_{S}\left(K_{S}+F\right) \rightarrow O_{F}\left(K_{F}\right) \rightarrow 0
$$

yields the long exact cohomology sequence

$$
0 \rightarrow H^{0}\left(S, \mathcal{O}_{S}\left(K_{S}\right)\right) \rightarrow H^{0}\left(S, \mathcal{O}_{S}\left(K_{S}+F\right)\right) \stackrel{r}{\longrightarrow} H^{0}\left(F, \mathcal{O}_{F}\left(K_{F}\right)\right) \rightarrow H^{1}\left(S, \mathcal{O}_{S}\left(K_{S}\right)\right) \rightarrow 0 .
$$

This implies $\operatorname{dim}(\operatorname{Im}(r))=1$; that is, the rational map determined by $\left|K_{S}+F\right|$ contracts the general curve in $|F|$ to a point. This is a contradiction, because the canonical image of $S$ is a surface.

Finally, assume that $\Sigma$ is a quadric cone. The same arguments as before show that the line ruling of $\Sigma$ determines a pencil $|F|$ on $S$ with $K \cdot F=4, F^{2}=2$. Then, as above, the rational map determined by $\left|K_{S}+F\right|$ contracts the general curve of $|F|$ to a point, again a contradiction, which shows that case (ii) cannot occur either. This finishes the proof of the proposition.

Remark 4.5. In order to exclude the existence of surfaces $S$ as in Claim 4.2 we could have argued, in principle, as in the proof of Theorem 3.2; that is, we could have used the results in [CMP14]. To do so, one should first prove that the general curve in $\mathcal{K}_{S}$ is smooth. This is not shorter, actually it is a bit more involved, than the proof presented here.

\section{REFERENCES}

ACGH85 E. Arbarello, M. Cornalba, P. Griffiths and J. Harris, Geometry of algebraic curves, Vol. I, Grundlehren der Mathematischen Wissenschaften 267, Springer-Verlag, New York, 1985.

AH91 D. Abramovich and J. Harris, Abelian varieties and curves in $W_{d}(C)$, Compositio Math. 78 (1991), 227-238.

Bea78 A. Beauville, Surfaces algébriques complexes, Astérisque 54, Soc. Math. de France, 1978.

Bea82 A. Beauville, L'inégalité $p_{g} \geqslant 2 q-4$ pour les surfaces de type général, Appendix to [Deb82] Bull. Soc. Math. de France, 110 (1982), 343-346.

Bea88 A. Beauville, Annulation du $H^{1}$ et systèmes paracanoniques sur les surfaces, J. reine angew. Math. 388 (1988), 149-157.

BH04 C. Birkenhake and H. Lange, Complex Abelian Varieties, Second edition, Grundlehren der Mathematischen Wissenschaften 302, Springer-Verlag, Berlin, 2004. http://dx.doi.org/ 10.1007/978-3-662-06307-1 


\section{Ciliberto, Mendes Lopes, Pardini}

BPvdV04 W. Barth, C. Peters and A. Van de Ven, Compact complex surfaces, Second Edition, Ergebnisse der Mathematik und ihrer Grenzgebiete 3, Springer-Verlag, Berlin, 2004. http://dx.doi. org/10.1007/978-3-642-57739-0

Cat81 F. Catanese, On a class of surfaces of general type, in Algebraic surfaces, (Varenna-Como, 1977), 269-284, Liguori, Naples, 1981.

CCM98 F. Catanese, C. Ciliberto and M. Mendes Lopes, On the classification of irregular surfaces of general type with non birational bicanonical map, Trans. Amer. Math. Soc. 350(1) (1998), 275-308.

CFM97 C. Ciliberto, P. Francia and M. Mendes Lopes, Remarks on the bicanonical map for surfaces of general type, Math. Z. 224 (1997), no. 1, 137-166.

CML02 C. Ciliberto and M. Mendes Lopes, On surfaces with $p_{g}=q=2$ and non-birational bicanonical maps, Adv. Geom. 2 (2002), 281-300.

CMP14 C. Ciliberto, M. Mendes Lopes and R. Pardini, Abelian varieties in Brill-Noether loci, Adv. Math. 257 (2014), 349-364. http://dx.doi.org/10.1016/j.aim.2014.02.024

Deb82 O. Debarre, Inégalités numériques pour les surfaces de type général, Bull. Soc. Math. de France 110 (1982), 319-342.

DF93 O. Debarre and R. Fahlaoui, Abelian varieties in $W_{d}^{r}(C)$ and points of bounded degree on algebraic curves, Compositio Math. 88 (1993), 235-249.

dFr05 M. de Franchis, Sulle superficie algebriche le quali contengono un fascio irrazionale di curve, Rend. Circ. Mat. Palermo, 20 (1905).

GL87 M. Green and R. Lazarsfeld, Deformation theory, generic vanishing theorems, and some conjectures of Enriques, Catanese and Beauville, Invent. Math. 90 (1987), 416-440.

Hor77 E. Horikawa, On algebraic surfaces with pencils of curves of genus 2, in Complex analysis and algebraic geometry, 79-90, Iwanami Shoten, Tokyo, 1977.

Hor82 E. Horikawa, Algebraic surfaces of general type with small $c_{1}^{2} V$, J. Fac. Sci. Univ. Tokyo Sect. IA Math. 28 (3) (1982) 745-755.

Man03 M. Manetti, Surfaces of Albanese general type and the Severi conjecture, Math. Nachr. 261/262 (2003), 105-122. http://dx.doi.org/10.1002/mana.200310115

MPP13 M. Mendes Lopes, R. Pardini and G.P. Pirola, Continuous families of divisors, paracanonical systems and a new inequality for varieties of maximal Albanese dimension, Geometry \& Topology 17 (2013), 1205-1223.

Par05 R. Pardini, The Severi inequality $K^{2} \geqslant 4 \chi$ for surfaces of maximal Albanese dimension, Invent. Math. 159, no. 3 (2005), 669-672.

Pen11 M. Penegini, The classification of isotrivially fibred surfaces with $p_{g}=q=2$, with an appendix by Sönke Rollenske, Collect. Math. 62 (2011), no. 3, 239-274.

Zuc03 F. Zucconi, Surfaces with $p_{g}=q=2$ and an irrational pencil, Canad. J. Math. 55 (3) (2003), 649-672.

Ciro Ciliberto cilibert@axp.mat.uniroma2.it

Dipartimento di Matematica, II Università di Roma, Italy

Margarida Mendes Lopes mmlopes@math.tecnico.ulisboa.pt

Departamento de Matemática, Instituto Superior Técnico, Universidade de Lisboa Av. Rovisco

Pais, 1049-001 Lisboa, Portugal

Rita Pardini pardini@dm.unipi.it

Dipartimento di Matematica, Università di Pisa, Largo B. Pontecorvo, 5, 56127 Pisa, Italy 